\title{
Application of Rural Residential Hourly Load Curves in Energy Modelling
}

\author{
N Cross and C. T. Gaunt, Member, IEEE
}

\begin{abstract}
This paper describes the methods used to determine hourly activity load curves for use in energy models. It explains how Conditional Demand Analysis curves have been used in combination with appliance penetration levels to develop appliance hourly load curves based on and representative of measured load data. It shows how these can be combined to determine hourly activity load curves, which contribute to the accuracy of energy models used for energy policy development and energy planning.
\end{abstract}

Index Terms--Data conversion, data models, load modelling, rural areas.

\section{INTRODUCTION}

$\mathrm{E}^{\mathrm{s}}$ NERGY models are useful in simplifying complex energy systems. The large number of variables, or components, involved in any energy system causes it to be virtually impossible to analyse without simplification. Models allow a complicated and involved energy system to be broken down into its essential parts using mathematical equations. They can thus assist engineers and decision-makers in understanding these systems, which ultimately improves planning and design processes. The very characteristics that make models useful, however, also ensure that they can never be completely accurate. Due to the fact that models are often linear and simplistic, they tend to offer a representation of a system that does not entirely reflect reality [1]. Models are also intensely data-dependent. Models that are sensitive to data only provide results that are meaningful and useful if the data fully reflects the actual scenario that is being studied. Ensuring that correct data representing reality is entered into the model is the task of the user.

More recent and advanced models, such as the optimisation model TIMES (The Integrated MARKAL Eprom System), allow a user to enter a system's energy demand data to a high resolution. In other words, it gives the user the opportunity to make use of time-differentiated curves to model the demand levels of a particular activity, for example hourly loads for cooking [1]. It follows that load curves are far better in modelling realistic energy demands than 'flat-rate' data. For

Both authors are in the Department of Electrical Engineering, University of Cape Town, South Africa. (e-mail: crossn@powerelec.uct.ac.za and ctg@eng.uct.ac.za). example, cooking may require $13 \mathrm{GJ}$ per household per year in a typical rural village, but this information alone provides no insight as to when this energy is needed. The model may thus choose to supply these needs at any time of the day, perhaps, for example, when electricity is least expensive, at three o'clock in the morning.

It is clear that a more detailed representation of reality is needed for modelling purposes. This paper explains how hourly load curves have been developed.

\section{PROBLEMS OF COLLECTING DATA FOR MODELLING}

Obtaining detailed and accurate data on energy systems is challenging, particularly for complex and developing systems such as those describing the household energy needs of rural villages. Meeting the need for accurate data is, in fact, one of the greatest obstacles in the modelling process. The reason for this is twofold.

First, little research has been conducted in the field of energy habits of rural households in Southern Africa and even the rest of Africa [2]. In South Africa specifically, only during the 1970s did the general focus of energy planning shift away from energy supply options towards the energy needs of people. The concept of integrated energy planning evolved when it became clear that the close relationship between energy supply and demand needed to be addressed throughout the planning process. Integrated energy planning requires an extensive database on the energy needs and habits (both energy and social) of the people in a relevant area. Before the 1990s, energy planning for rural areas was not a priority of the South African government . Although a few energy research projects carried out in the $70 \mathrm{~s}, 80$ s and early 90 s considered the energy needs of rural households, they were not extensive enough to develop a substantial and relevant database [2].

Secondly, it is almost impossible to keep track of the habits of low-income non-electrified rural households. The only data available on poor, rural, non-electrified villages is that which has been obtained in surveys and questionnaires. No survey has been detailed enough to produce data that could be used to develop an hourly activity load curve for a particular village. And even if surveys were to pose questions of that nature, the sample size used in the survey may lead to inaccurate load curves. One of the only methods that would enable accurate rural activity load curves to be developed is the recording of the time and amount of fuel used during a particular activity. This is an unreasonable task to expect of anyone, especially 
rural villagers who may be uneducated or illiterate.

In fact, it is also difficult to keep track of the habits of newly-electrified low-income rural households. Methods have been developed to collect representative time-based electrical load data from communities of residential customers using data loggers installed on the network. Extensive data has been collected from urban and rural communities of households with high, medium and low income. Together with surveys of demographic and social parameters, the load data provides models of electricity use in communities of customers under various conditions [3], [4]. However, the electricity may not be the only or primary form of energy. Research has shown that electricity does not immediately become the primary fuel of a newly-electrified rural household for various reasons, including costs and tradition [5], [6]. Thus, the question remains: how does a user obtain or develop the hourly activity load curves of a low-income rural household?

\section{DEVELOPING HOURLY ACTIVITY LOAD CURVES FOR A RURAL VILLAGE}

The seemingly contradictory solution is to use data from specific electrified households, even if the system that is being modelled is a non-electrified rural village. Electrical load curves from an electrified village can be used as an indication of the total energy load curves of particular activities. This method may not be completely accurate because electrified villages do not necessarily use only electricity to meet all their energy needs. Nonetheless, it is the only method available and it is useful in the modelling process.

Specific activity (electrical) load curves are not available for any of the electrified villages in which energy modelling research is being done, but conditional demand analysis (CDA) curves can be used to develop them [7], [8]. CDA curves have been derived from surveys conducted in, and the logging of, 15 rural villages [9]. When electrical data is logged (this automatically implies that the data can be collected only from electrified villages), it can only provide overall load curves of a village. In other words, the total electricity consumption of each household is logged on a regular basis. In conjunction with this data logging, market researchers record specific information about the houses that were logged. This information includes details such as the size of the house, the number of occupants in the house, and the number and type of electrical appliances that the household uses. Mathematical methods such as cross-correlation then allow the many averaged load curves to be disaggregated into several appliance profiles based on the information gathered by the market researchers [3]. These appliance profiles can then be used as general curves for any community with characteristics similar to the villages that were analysed.

The main difficulties in using these CDA curves to develop appliance curves for a specific village lie in finding CDA curves that are appropriate, and then determining the penetration levels of the relevant village. The points on the CDA curves are coefficients with units in amps, and cannot be used as they are. These coefficient values need to be multiplied by the appliance penetation levels of the relevant community to give a curve that is applicable. They can also be modified to have different units, for example kVA.

The penetration level of a particular population refers to the proportion of that population that uses a specific appliance. This level varies with income and time electrified. These factors need to be taken into consideration in order to obtain realistic penetration levels. Often, surveys show penetration levels at a particular (mostly present) time and income level, and these can be extrapolated by making assumptions regarding income growth. Unfortunately, income growth in rural areas can be extremely unpredictable and stagnant. The method used to determine penetration curves and levels is described in the next section.

The CDA curves are general averages that will never be entirely accurate, but are one of the useful tools that can be used when other data is unavailable. Once appliance curves have been developed using the C.DA curves and penetration levels, these can be combined and used to develop the total activity curves (e.g. cooking). These activity curves can then be used to specify the demand for the relevant energyconsuming activities. This approach is illustrated in the flow diagram in the fig. 1

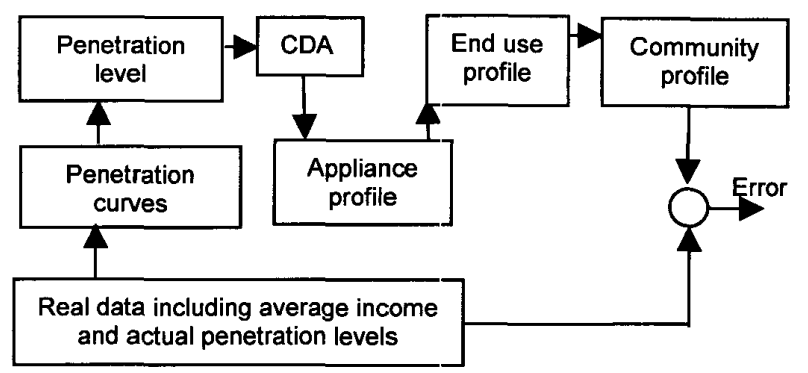

Fig. 1 Method to determine hourly activity load curves

\section{METHOD TO DETERMINE PENETRATION CURVES OF APPLIANCES}

In all energy models, residential energy demand is described in terms of activities performed in the household. These include cooking, space heating, water heating, lighting, refrigeration and 'other', which includes entertainment and communication activities [2]. Each of these activities needs to be expressed in terms of an hourly load curve. The hourly electrical load curves of specific appliances can be used to estimate the total energy load curve for corresponding activities. The load curves of hotplates, electric stoves and microwaves could be combined to obtain a cooking profile. The load curve of electric heaters could be used as a space heating profile, and so on. Thus the load curves of these specific appliances are needed. The process of determining these begins, as described, with shaping the penetration curves of the same appliances.

As mentioned, penetration levels depend on two dominant factors: time electrified and income. Available data offers 
general penetration as a function of income (and income growth), and as a function of time electrified. For the latter, three different scenarios are considered: the appliance penetration levels at 7-12 months, 3-4 years, and 10 years after electrification. This general data is used to develop penetration profiles at each period of electrification as a function of income. One set of profiles is shown in Fig. 2.

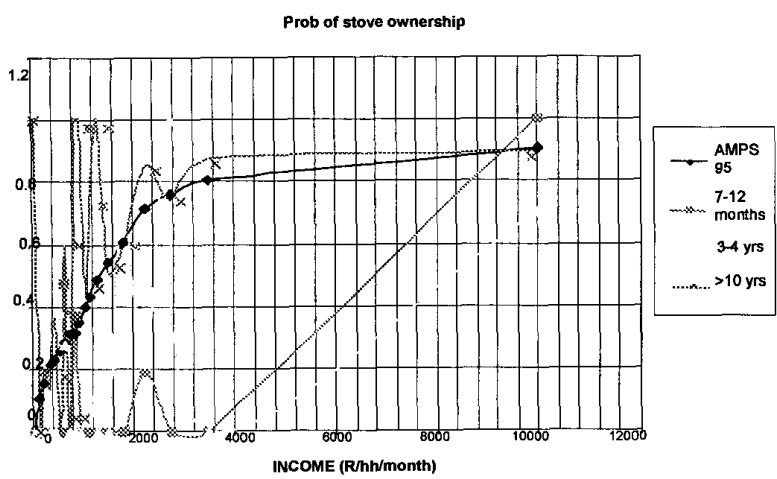

Fig. 2 Penetration profiles as a function of income

A number of these curves are fairly 'rollercoaster' in nature, because of the small sample sizes that were used in some years for the construction of the original general appliance penetration curves. A fourth curve labelled 'AMPS 95' is also featured on these curves. This is a 'smoothed' curve developed by the AMPS group, and shows the penetration levels as a function of income only (this was put in as a gauge with which to compare the more erratic graphs obtained directly from survey data). The AMPS data was collected through surveys conducted by Telmar Communications Services.

At this stage the curves are smoothed out manually to present more realistic appliance penetration rates. 'Real' data on the community (or a similar electrified village) being studied must be available to provide the average income of the households in that community, and the penetration levels of a number of electrical appliances for a particular year. An average income growth rate (which can be obtained from the households surveys for the sampled communities and from the national census) is then used in conjunction with the real data (which acts as a reference) to convert the curves (functions of income) to a single curve representing appliance penetration as a function of year. An example of a single, smooth curve is shown in fig. 3 .

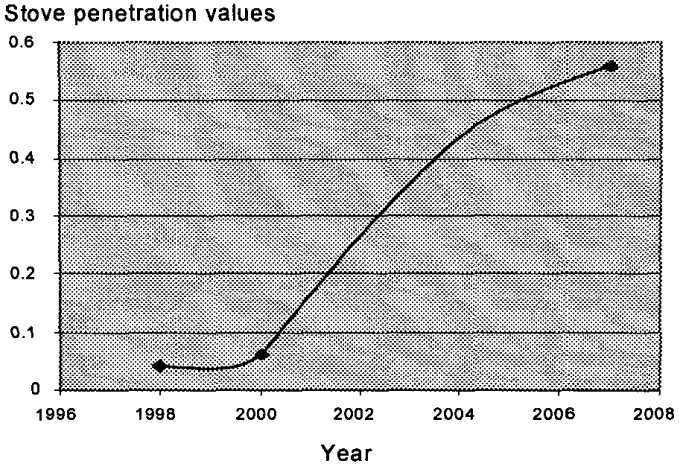

Fig. 3 Appliance penetration as a function of time

Finally, the penetration levels are multiplied by the CDA coefficients on a year-to-year basis which results in appliance profiles for a number of years. The hotplate, stove and microwave profiles can be combined to give a cooking profile, while other activity profiles are based on the load curves of a single appliance. A final cooking profile according to time of day is shown in Fig. 4.

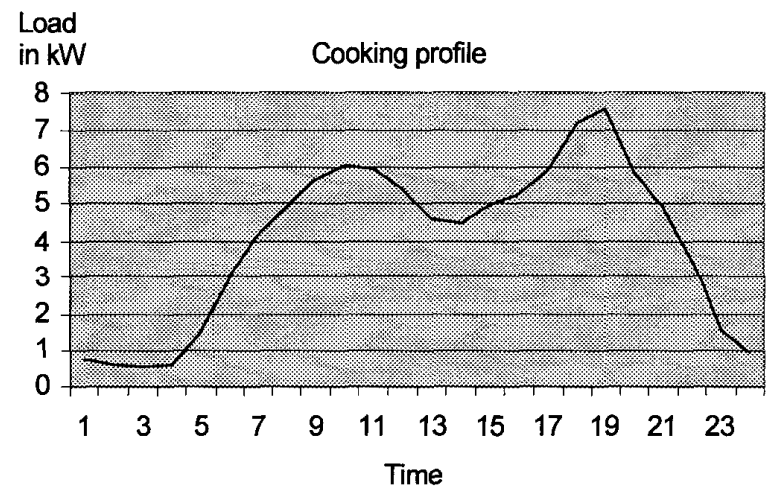

Fig. 4 Activity profile as a function of time of day

\section{APPLICATION OF ACTIVITY CURVES IN MODELS IN A BROADER CONTEXT}

The method discussed in this paper has been used to develop an extensive TIMES models. TIMES is an optimisation model which uses technology databases containing detailed information on the intended area of application and the relevant cost aspects involved. Although optimisation models are flexible, a high level of detail often needs to be incorporated into the models for the simulation to be realistic. They are very useful tools in the policy-making and planning and design processes, because they are able to indicate an optimal solution to a specific problem or system. For example, an optimisation model may offer a least-cost scenario that will meet specific needs within certain constraints. This has enormous economic benefits. Another application of these models is the prediction of the behaviour 
of energy users. They may show that in the case of a village not having access to grid electricity, diesel generators might be used extensively, given the assumption of other appropriate conditions.

In 2002, a TIMES model was developed to obtain a leastcost solution to meeting the energy needs of a typical rural, non-electrified village in South Africa. This project was carried out by the Energy Research Institute in conjunction with the Program on Energy and Sustainable Development (PESD) associated with Stanford University [10]. The aim of this research was to develop a comprehensive and accurate model that could be effectively used in the planning of rural electrification. Although the data in the model was not based entirely on an actual rural village, the load curves describing the demand data of the village were based entirely on the methods set forward in this paper, together with load and appliance data from a real village. The resulting model offers a range of scenarios and results. For example, the 'base' scenario, which determined the probable costs and behaviour of the system if all of its features (besides population) remained the same over time, found that traditional fuels were still the cheapest supply option for cooking, space- and waterheating. Another scenario looked at the likely costs and results if only isolated generation were available to the village. The benefits of such a model are impressive, but only if the results are accurate enough to use in the planning process. The aim of the methods described in this paper is to ensure that models are as accurate, and therefore useful, as possible.

\section{CONCLUSIONS}

In many modelling processes, data collection can be extremely difficult, leading to significant gaps in a required database and the making of many assumptions. Due to the fact that little data is available on the energy habits of rural villagers, modelling of the energy habits of such communities requires data development. The development of data is significant in the modelling process due to the intrinsic dependence of models on accurate and detailed data. One of the weaknesses of energy models is that the results and solutions they provide may be inaccurate, and therefore of little use, if the data entered into the model is not accurate and detailed. It is especially difficult for a user to obtain accurate and detailed data on developing energy systems, such as a rural community. In such a case, the ability to develop data becomes significant because 'true' data is simply not available.

CDA curves are general hourly load curves that have been obtained through the analysis of the energy data of a number of rural communities. When disaggregated into 'applianceprofile' form, they can be used in combination with appliance penetration levels to obtain hourly appliance load curves. The penetration levels need to be based on 'real' data from the system that is being studied and representative data from general surveys. These can then be combined to determine hourly activity load curves, which can improve the accuracy of the demand data in rural energy models. This detailed data will significantly improve the relevance of the results obtained from the energy model. These results can then be used directly in the planning and decision-making processes.

\section{ACKNOWLEDGMENT}

The authors gratefully acknowledge the contribution by $\mathbf{M}$. Dekenah of data from the National Load Research Project, supplied for this analysis.

The modelling work was carried out as part of a project in the Energy Research Institute, University of Cape Town.

\section{REFEFENCES}

[1] J. Harnisch, M. Koch, N. Hohne, K. Blok, "Prospects for the application of energy models in the design of climatic policies", 6th Greenhouse Gas Control Technologies Conference, International Energy Agency, Japan, 2002

[2] Y. Afraane-Okese, "Domestic energy' use database for integrated energy planning", Energy and Development Research Centre, University of Cape Town, 1998.

[3] M. Dekenah, S. Heunis, "Results of the NRS LR project 1998." Proceedings Domestic Use of Electrical Energy Conference, Cape Town, p94-98. 1999.

[4] C. T. Gaunt, R. Herman, M Dekenah, R L Sellick, S. W Heunis, "Data collection, load modelling and probabilistic analysis for LV domestic electrification." International Conference on Electricity Distribution (CIRED), Nice, June, 1999.

[5] T. Williams, "Energy supply options for low-income urban households", Energy and Development Research Centre, University of Cape Town 1994

[6] G. Prasad, Personal Communication, Energy and Development Research Centre, 2002-11-14

[7] M. Parti, C. Parti, "The total and appliance specific conditional demand for electricity in the household section", Journal Economics, No $11, p$ 309-324

[8] H. Nyikos, "Aggregation of whole house load into its various components (Conditional Demand Analysis)". Proceedings Domestic Use of Electrical Energy, Cape Town, p170-174, 1999

[9] M. Dekenah, Personal communication, 2002-11-14

[10] M. Howells, T. Alfstadt, N. Cross, L. Jeftha, "Rural Energy modelling", Rural energy transitions meeting, Program on Energy and Sustainable Development, India, 2002.

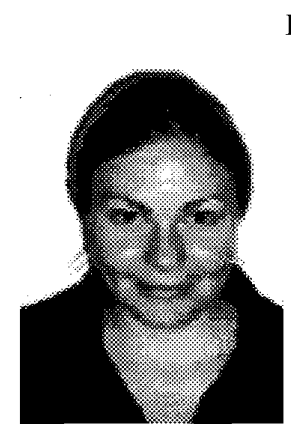

\section{BIOGRAPHIES}

Nicola Cross graduated from University of Cape Town in 2002 with BSc(Eng). She has participated in the TIMES project, a collaborative energy modelling project between Stanford University and UCT, and also spent a short period at University of Stuttgart working on modelling. She is presently a full-time postgraduate student working in distribution systems engineering at UCT.

Trevor Gaunt is an Associate Professor. He has 30 years of experience in manufacturing, consulting engineering and research. 